\title{
Early endocrine alterations reflect prolonged stress and relate to 1-year functional outcome in patients with severe brain injury
}

\author{
Djordje Marina, Marianne Klose, Annette Nordenbo ${ }^{1}$, Annette Liebach ${ }^{1}$ \\ and Ulla Feldt-Rasmussen
}

Department of Medical Endocrinology PE2131, Rigshospital, Copenhagen University Hospital, Copenhagen, Denmark and ${ }^{2}$ Traumatic Brain Injury Unit, Department of Neurorehabilitation, Glostrup Hospital, Copenhagen University Hospital, Glostrup, Denmark
Correspondence should be addressed to U Feldt-Rasmussen Email

ufeldt@rh.dk

\begin{abstract}
Objective: Severe brain injury may increase the risk of developing acute and chronic hypopituitarism. Pituitary hormone alterations developed in the early recovery phase after brain injury may have implications for long-term functional recovery. The objective of the present study was to assess the pattern and prevalence of pituitary hormone alterations 3 months after a severe brain injury with relation to functional outcome at a 1-year follow-up.

Design: Prospective study at a tertiary university referral centre.

Methods: A total of 163 patients admitted to neurorehabilitation after severe traumatic brain injury (TBI, $n=111)$ or non-TBI $(n=52)$ were included. The main outcome measures were endocrine alterations 3.3 months (median) after the brain injury and their relationship to the functioning and ability of the patients at a 1-year follow-up, as measured by the Functional Independence Measure and the Glasgow Outcome Scale-Extended.

Results: Three months after the injury, elevated stress hormones (i.e. 30 min stimulated cortisol, prolactin and/or IGF1) and/or suppressed gonadal or thyroid hormones were recorded in 68 and $32 \%$ of the patients respectively. At 1 year after the injury, lower functioning level (Functional Independence Measure) and lower capability of performing normal life activities (Glasgow Outcome Scale-Extended) were related to both the elevated stress hormones $(P \leq 0.01)$ and the reduced gonadal and/or thyroid hormones $(P \leq 0.01)$ measured at 3 months.

Conclusion: The present study suggests that brain injury-related endocrine alterations that mimic secondary hypogonadism and hypothyroidism and that occur with elevated stress hormones most probably reflect a prolonged stress response 2-5 months after severe brain injury, rather than pituitary insufficiency per se. These endocrine alterations thus seem to reflect a more severe disease state and relate to 1-year functional outcome.
\end{abstract}

European Journal of

Endocrinology

(2015) $172,813-822$

\section{Introduction}

Acquired traumatic brain injury (TBI) and non-TBI are recognised as major critical health problems worldwide (1), and health authorities expect TBI to become the leading cause of death and disability by the year 2020 (2). Both TBI and non-TBI may be complicated by short- and long-term pituitary dysfunction, which has possible implications for the recovery and functional outcomes of patients (3). The reported prevalence of acute and chronic
(C) 2015 European Society of Endocrinology Printed in Great Britain hypopituitarism after TBI and non-TBI has varied considerably between studies, ranging from 13 to $56 \%$ in the acute phase after TBI $(4,5,6)$ and from negligible to well above $50 \%$ in the chronic phase $(4,5,6,7,8,9)$. Such discrepancies probably reflect differences in study populations and diagnostic set-up, as has been recently discussed $(8,10)$. Also, non-TBI conditions, such as aneurysmal subarachnoid haemorrhage (11),

Published by Bioscientifica Ltd. 
chronic subdural haematoma (12) and ischaemic stroke (13), have been related to an increased prevalence of hypopituitarism in some studies but not in others $(14,15)$.

Previous assessments of the relationship between post-traumatic hypopituitarism and outcomes have shown conflicting results. Some have suggested that hypopituitarism was related to increased disability (16) and a decreased quality of life $(16,17)$, whereas others have suggested that quality-of-life deficits were more closely associated with intracerebral haemorrhagic lesions than with hypopituitarism per se (18). Peak growth hormone $(\mathrm{GH})$ has been shown to be an independent predictor of poorer outcomes, as measured by rehabilitation scales that evaluate cognition and disability (19), but such relations were recently questioned (20). Reduced testosterone concentrations were also recently described as being related to worse functional outcomes in TBI patients $(21,22)$, which led the authors to suggest that secondary hypogonadism may limit the pace of recovery during neurorehabilitation.

Based on early reports that suggested a high risk of post-traumatic hypopituitarism, a screening programme was introduced to assess pituitary function at the Department of Neurorehabilitation's Traumatic Brain Injury Unit at Copenhagen University Hospital, Glostrup. We hypothesised that the results from pituitary hormone assessment in the post-acute phase (i.e. $\sim 3$ months postinjury) would relate to functional and cognitive outcomes at 1-year follow-up.

\section{Subjects and methods}

\section{Subjects}

In Denmark, the post-acute rehabilitation of patients with the most severe TBI and non-TBI is centralised at two hospitals, each of which covers $\sim 50 \%$ of the Danish population of 5.5 million. Highest priority for admission is given to the most severely brain-injured patients, who are defined as having a Glasgow Coma Scale score of 3-12 in the first days after the cessation of sedation. Patients with a Glasgow Coma Scale score of 12-14 in the first days after the cessation of sedation are also admitted if they have severe focal neurological deficits or are severely agitated.

From 1st June 2007 until 31st May 2011, a total of 272 patients $\geq 15$ years old were admitted to the Department of Neurorehabilitation's Traumatic Brain Injury Unit in Copenhagen. Patients with known pituitary disease or clinically significant renal disease or who were pregnant or breast-feeding were not considered for inclusion. Twelve patients died, and 51 were discharged to home within 2 months of their initial admission to neurorehabilitation without pituitary testing for unknown reasons. Pituitary assessment was also not performed in 46 patients who had longer stays at the Neurorehabilitation Unit (in 15 patients because of severe agitation and in 31 patients for unknown reasons). Thus, we included 163 patients who had available pituitary hormone measurements: 111 TBI and 52 non-TBI patients. The non-TBI patients included 28 with subarachnoid, intracerebral and non-traumatic haemorrhage, nine with anoxic brain injury, four with occlusion or stenosis of cerebral arteries not causing cerebral infarction, three with brain inflammation (meningitis), two with cerebral oedema, two with sepsis and one each with cerebral infarction, arteriovenous malformation, traumatic fat embolism and central pontine myelinolysis.

The study was approved by the Local Ethical Committee (J.nr. H-D-2009-054) and the Danish Data Protection Agency (J.nr. 30-0831). According to the Danish Act on Processing of Personal Data, a registry study without contact to the persons involved does not require verbal or written consent.

\section{Endocrine function testing and diagnostic criteria for endocrine alterations}

Pituitary function testing was performed at a median of 3.3 months (range 2.1-4.9) after the brain injury and related to 1-year outcome data of functioning and ability as part of routine assessment in the department. Hormone assessment was performed between 0800 and $1300 \mathrm{~h}$, and it included baseline measurements of follicle-stimulating hormone (FSH), luteinising hormone (LH), testosterone (males), oestrogen (females), thyroid-stimulating hormone $(\mathrm{TSH})$, free thyroxine $\left(\mathrm{FT}_{4}\right)$, prolactin and insulin-like growth factor 1 (IGF1). The hypothalamicpituitary-adrenal axis was assessed using an adrenocorticotrophic hormone (ACTH) stimulation test that measured cortisol concentrations at baseline and $30 \mathrm{~min}$ after the i.v. administration of $250 \mu \mathrm{g}$ Synacthen (Defiante Farmacêutica S.A., Funchal, Madeira-Portugal). If patients presented with thirst and/or increased urine volumes, diabetes insipidus was assumed and vasopressin therapy was initiated.

Insufficiencies were defined in relation to local sex- and age-related reference ranges. Secondary hypogonadism in postmenopausal women was defined as inappropriately low gonadotrophins (FSH and $\mathrm{LH}$ ) for age, in premenopausal women as the presence of 
amenorrhoea or oligomenorrhoea associated with low oestrogen and inappropriately low gonadotrophins and in men as low testosterone associated with inappropriately low gonadotrophins. Secondary hypothyroidism was defined as subnormal $\mathrm{FT}_{4}$ concentration associated with an inappropriately low TSH concentration. Normal reference ranges for the Immulite assay for serum cortisol was based on 100 healthy controls, with reference intervals given as the 2.5 th and 97.5 th percentiles (23). Thus, adrenal insufficiency was defined as $<500 \mathrm{nmol} / 1$ 30 min cortisol in response to the Synacthen test, and elevated basal cortisol was described as concentrations of $>665 \mathrm{nmol} / \mathrm{l}$ and stimulated cortisol as concentrations of $>841 \mathrm{nmol} / \mathrm{l}$.

Three of the 52 female patients had been using contraceptive pills, which were discontinued at least 8 weeks prior to endocrine evaluation. At the time of endocrine evaluation, 12 patients were being treated with antipsychotics, 18 with antidepressants, 23 with antiemetics and 37 with antiepileptic drugs (the majority using Gabapentin and Fenytoin $(n=19)$, a minority using Lamotrigin $(n=2)$ and 16 using other antiepileptic medications).

\section{Function and ability assessments}

Outcome measures of functioning and ability were assessed at admission and discharge from neurorehabilitation and at a 1-year follow-up.

The Functional Independence Measure is a scale consisting of 18 items describing various activities in daily living. These 18 items are further divided into physical (13 items) and cognitive domains (five items). Each item is rated on a seven-point scale from 1 (total assistance needed) to 7 (full independence). The added scores of the 18 items give a minimum total score of 18 and a maximum score of 126 . For practical purposes, the overall total scores were divided into three subgroups that indicated the assistance required in order for the individual to carry out daily activities: complete dependency (18-55), moderate dependency (56-74) and low-level dependency to complete independency (75-126). Further, the Functional Independence Measure score can be divided into a motor score, with a maximum of 91 , and a cognitive score, with a maximum of 35 . The Functional Independence Measure was performed at admission to neurorehabilitation, at discharge and at the 1-year follow-up.

The Glasgow Outcome Scale-Extended is an eightpoint scale used to describe the actual functioning of the patient in the period after the brain injury; it refers to independence at and outside the home, different social activities, work capability and return to normal life. For practical purposes, it is divided into two groups: lower scores range from 1 (coma) to 4 (upper severe disability), and higher scores range from 5 (lower moderate disability) to 8 (upper good recovery). Patients with scores from 1 to 4 are more dependent on assistance, whereas patients scoring between 5 and 8 need less assistance performing activities at and outside the home. The Glasgow Outcome Scale-Extended was performed at discharge from neurorehabilitation and at the 1-year follow-up.

In addition, data on post-traumatic amnesia was assessed in the TBI patients using the Galveston Orientation and Amnesia Test. The period of post-traumatic amnesia was defined as the number of days between the date of the TBI and the date when the patient was out of the post-traumatic confused state. Post-traumatic amnesia that lasted for more than 4 weeks indicated a very severe TBI.

\section{Assays}

The type and performance characteristics of the assays used for the hormone measurements are shown in Supplementary Table 1, see section on supplementary data given at the end of this article.

\section{Statistical analyses}

Categorical data are presented as $n(\%)$, continuous as mean ( \pm s.D. $)$ if normally distributed and otherwise as median (range).

Categorical data were compared by a $\chi^{2}$ test. Cramer's $V$ coefficient was used to evaluate associations between two nominal variables. Between-group comparisons of normally distributed continuous data were analysed by Student's $t$-test or by Mann-Whitney $U$ test. Regression analyses were conducted to analyse the relation between outcome measures (Functional Independence Measure and Glasgow Outcome Scale-Extended) and dependent covariates (injury characteristics, medication, including analgesics, antidepressants and antiepileptic drugs, and hormonal hypo/hyper secretion). Basal and stimulated cortisol concentrations of the patients were compared to 100 healthy persons in which a Synacthen test was performed and cortisol was measured by Immulite assay (23).

Associations were performed on the pooled data from the TBI and non-TBI groups, because no significant difference was observed in the prevalence of hormone alterations between the groups. A difference was 
Table 1 Baseline characteristics of the included patients with traumatic brain injury (TBI) and non-TBI, with additional data on hormonal assessment. Data are given as number (\%) or mean (s.D.)/median (range, 10-90\%).

\begin{tabular}{l}
\hline \\
Gender, men (\%) \\
Age at admission (years) \\
Glasgow Coma Scale score at admission \\
Time from trauma to neurorehabilitation (days) \\
Length of neurorehabilitation (days) \\
CT/MR finding (TBI) \\
Traumatic subarachnoid haemorrhage \\
Cranial fracture \\
Shearing lesions \\
Cerebral oedema \\
Cause of injury (non-TBI) \\
Subarachnoid haemorrhage \\
Intracerebral haemorrhage \\
Anoxic brain injury \\
Other \\
Functional Independence Measure score at admission \\
Post traumatic amnesia, days (\%) \\
$>4$ weeks \\
$<4$ weeks \\
Number of patients with pituitary assessment (\%) \\
Synacthen test, including basal cortisol \\
Prolactin \\
Insulin-like growth factor 1 (IGF1) \\
Gonadal hormones \\
Free thyroxine (FT 4 ) \\
Median concentration (range) of hormonal measurement \\
Stimulated 30 min cortisol \\
Prolactin \\
IGF1 \\
Testosterone (males) \\
FT 4 \\
\end{tabular}

$\mathrm{CT}$, computerized tomography; MR, magnetic resonance.

considered significant when $P<0.05$. Statistical analyses were performed by SAS version 9.1 (SAS Institute, Inc., Cary, NC, USA).

\section{Results}

\section{Patient characteristics}

The patients' characteristics at admission are presented in Table 1 . The Synacthen test results were missing for 12 patients $(7 \%)$, whereas other hormonal measurements were missing in a minor proportion of the patients (Table 1). Patients who fulfilled the inclusion criteria but were not included $(n=109)$ did not differ from those included $(n=163)$ in terms of sex $(P=0.66)$ and age $(P=0.67)$, but they were less affected, as assessed by their shorter post-traumatic amnesia lengths $(P=0.001)$, higher Glasgow Coma Scale scores at admission $(P=0.03)$ and

\begin{tabular}{|c|c|}
\hline TBI & Non-TBI \\
\hline 111 & 52 \\
\hline $83(75 \%)$ & $28(54 \%)$ \\
\hline $42.7(18.5)$ & $47.9(16.0)$ \\
\hline $10.2(3.1)$ & $11.3(2.3)$ \\
\hline $20.7(13.0)$ & $28.6(18.6)$ \\
\hline $113.1(67.2)$ & $93.7(40.5)$ \\
\hline $64(57 \%)$ & / \\
\hline $42(38 \%)$ & \\
\hline $30(27 \%)$ & \\
\hline $26(23 \%)$ & \\
\hline \multirow[t]{4}{*}{ I } & $16(31 \%)$ \\
\hline & $12(23 \%)$ \\
\hline & $9(17 \%)$ \\
\hline & $15(29 \%)$ \\
\hline $18(18-44)$ & $18(18-25)$ \\
\hline $98(88 \%)$ & l \\
\hline \multicolumn{2}{|l|}{$13(12 \%)$} \\
\hline $102 / 111(92 \%)$ & $49 / 52(94 \%)$ \\
\hline 109/111 (98\%) & $50 / 52(96 \%)$ \\
\hline $109 / 111(98 \%)$ & $50 / 52(96 \%)$ \\
\hline 109/111 (98\%) & $50 / 52(96 \%)$ \\
\hline $110 / 111(99 \%)$ & $50 / 52(96 \%)$ \\
\hline $773 \mathrm{nmol} / \mathrm{l}(566-1240)$ & $774 \mathrm{nmol} / \mathrm{l}(491-1280)$ \\
\hline $0.61 \mathrm{nmol} / \mathrm{l}(0.15-4.9)$ & $0.59 \mathrm{nmol} / \mathrm{l}(0.16-10.6)$ \\
\hline $229 \mu \mathrm{g} / \mathrm{l}(64-486)$ & $222 \mu \mathrm{g} / \mathrm{l}(28-461)$ \\
\hline $12 \mathrm{nmol} / \mathrm{l}(1.0-28.0)$ & $9.9 \mathrm{nmol} / \mathrm{l}(0.2-17.5)$ \\
\hline $14.2 \mathrm{pmol} / \mathrm{l}(6.9-19.1)$ & $14.7 \mathrm{pmol} / \mathrm{l}(9.7-26.4)$ \\
\hline
\end{tabular}

better Functional Independence Measure total, physical and cognitive scores one year after admission (all $P=0.02$ ).

\section{Prevalence of endocrine alterations}

The prevalence of hormonal alterations at a median of 3.3 months after brain injury in the TBI and non-TBI patients is shown in Fig. 1. There were no significant differences in the prevalence of endocrine alterations between the TBI and non-TBI patients. The upper six panels in Fig. 1 demonstrate possible pituitary deficiencies. Hormonal alterations similar to secondary hypogonadism ( $24 \%$ of the TBI patients and $22 \%$ of the non-TBI patients) and hypothyroidism ( $9 \%$ of the TBI patients and $16 \%$ of the non-TBI patients) were the most commonly observed, whereas other deficiencies were observed in up to $5 \%$ of the TBI and non-TBI patients. The lower four panels in Fig. 1 show the prevalence of elevated stress hormones 


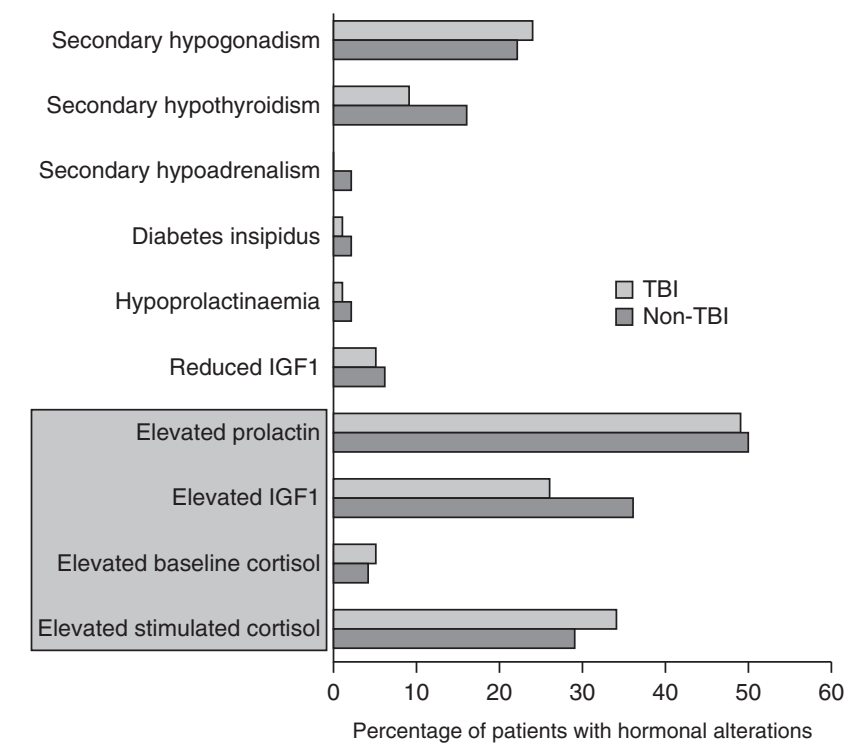

Figure 1

Percentage of patients with hormonal alterations presented separately in traumatic brain injury (TBI) and non-TBI groups. The shaded area represents the prevalence of elevated stress hormones.

(i.e. concentrations above the upper normal reference limit), including elevated prolactin (49\% of the TBI patients and $50 \%$ of the non-TBI patients), IGF1 (26\% of the TBI patients and $36 \%$ of the non-TBI patients), basal cortisol (5\% of the TBI patients and $4 \%$ of the non-TBI patients) and $30 \mathrm{~min}$ cortisol (34\% of the TBI patients and $29 \%$ of the non-TBI patients).

The study shows that $32 \%$ of the patients had suppressed gonadal or thyroid function (1\% were suppressed on both axes), whereas $68 \%$ had elevated stimulated 30 min cortisol, prolactin or IGF1 (29\% had an elevated concentration of more than one hormone). Elevated stress hormones and suppressed gonadal and/or thyroid function were concomitantly registered in 40 out of 151 (26\%) of the patients. Hyperprolactinaemia was related to suppressed thyroid function $(P=0.04$; Cramer's $V=0.17)$ but not to suppressed gonadal function $(P=0.67$; Cramer's $V=-0.03)$. Elevated stimulated $30 \mathrm{~min}$ cortisol was related to suppressed gonadal function $(P=0.02$; Cramer's $V=0.19)$ but not to suppressed thyroid function $(P=0.09$; Cramer's $V=-0.13)$.

\section{Functional Independence Measure}

Low median Functional Independence Measure total, motor and cognitive scores at admission were in agreement with very severely injured patients that had a high degree of dependency. Functional Independence Measures improved significantly during the rehabilitation period in both the TBI and non-TBI patients (Fig. 2). Thus, significantly fewer patients had a high degree of dependency at the 1-year follow-up as compared to baseline (i.e. at admission to neurorehabilitation) in both the TBI and non-TBI patients $(P<0.01)$ (Table 2$)$. In the TBI group, Functional Independence Measure scores were missing for 18 patients at the 1-year follow-up. In the non-TBI group, Functional Independence Measure scores were missing for one patient at admission, two patients at discharge and 12 patients at the 1-year follow-up visit (Table 2).

\section{Glasgow Outcome Scale-Extended}

At discharge, $72 \%$ of the TBI patients and $86 \%$ of the non-TBI patients had a very low Glasgow Outcome Scale-Extended score, which indicated a low degree of independent activity (Table 2). This was ameliorated at the 1-year follow-up visit in a significant number of patients in the TBI group $(P<0.05)$. In the TBI group, Glasgow Outcome Scale-Extended scores were missing for 18 patients at the 1-year follow-up (Table 2). In the non-TBI group, Glasgow Outcome Scale-Extended scores were missing for one patient at discharge and 12 patients at the 1-year follow-up (Table 2).

\section{Relationship between early endocrine alterations and functional outcome measures at the 1-year follow-up}

Worse Functional Independence Measure total, cognitive and physical scores at 1 year were related to lower Glasgow Coma Scale scores at referral and a need for longer rehabilitation $\left(P<0.001 ; \beta_{\text {total }}<-0.23 ; \beta_{\text {cognitive }}\right.$ $<-0.05 ; \beta_{\text {physical }}<-0.16$ ) but not to shearing lesions or medical treatment with antiepileptic, antidepressant or analgesic drugs (all $P>0.22$ ). Worse Functional Independence Measure scores were also related to secondary hypogonadism $\left(P \leq 0.02 ; \beta_{\text {total }}<-20 ; \beta_{\text {cognitive }}<-4.5\right.$; $\left.\beta_{\text {physical }}<-14\right)$ and increased stress hormones $(P \leq 0.02$; $\left.\beta_{\text {total }}<-13 ; \beta_{\text {cognitive }}<-4 ; \beta_{\text {physical }}<-15\right)$. Both secondary hypogonadism $(P=0.02 ; \beta=16)$ and increased stress hormones $(P=0.02 ; \beta=14)$ remained independently related to worse Functional Independence Measure scores in multivariate analyses after adjusting for the length of rehabilitation needed $\left(P \leq 0.01 ; R^{2}=0.23\right)$.

A low capability for returning to normal life activities, as indicated by a low Glasgow Outcome-Extended score at 1 year, was more frequent in patients with a lower Glasgow Coma Scale score at referral and a need for longer 


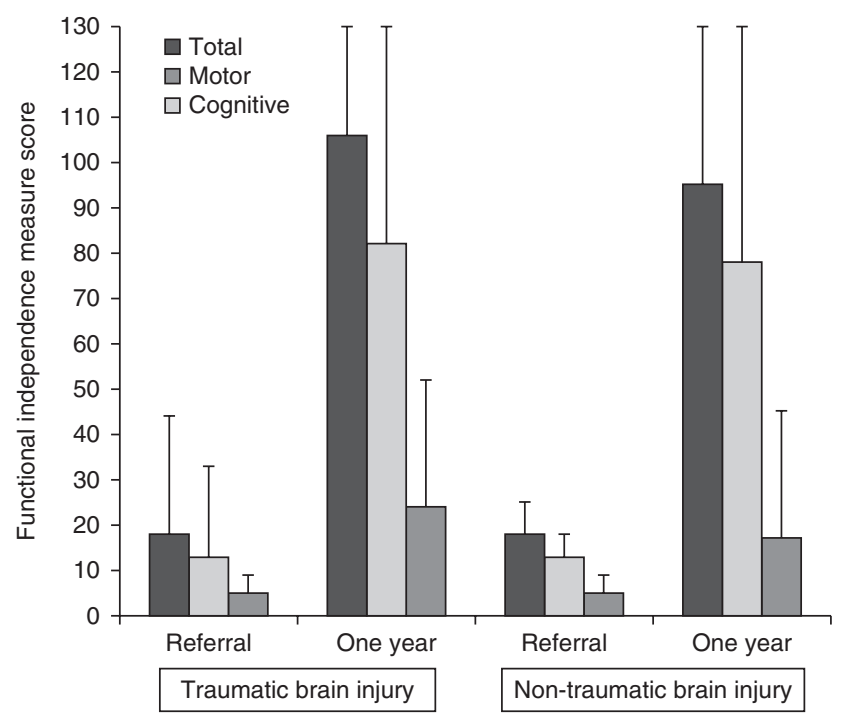

Figure 2

Median Functional Independence Measure scores presented with 10th and 90th percentile Cls of included patients with traumatic brain injury (TBI) and non-TBI at referral and at follow-up. Scores are divided into total, motor and cognitive scores. A Functional Independence Measure total score of 18 indicates that the patient was in need of total assistance, and a score of 126 suggests that the patient was fully independent. Maximum Functional Independence Measure scores, which are designated as optimal when the patient is completely independent, were: 126 (total), 91 (motor) and 35 (cognitive).

rehabilitation $(P<0.001)$ but not to shearing lesions or medical treatment with antiepileptic, antidepressant or analgesic drugs (all $P>0.32$ ). A low Glasgow OutcomeExtended score was also related to reduced gonadal and/or thyroid hormones $(P=0.04$; Cramer's $V=-0.17)$ and elevated stress hormones $(P=0.006$; Cramer's $V=-0.23)$. In multivariate analyses, adjusting for the length of rehabilitation needed increased stress hormones $(P=0.01)$ but not secondary hypogonadism $(P=0.17)$, which remained independently related to a worse Glasgow Outcome-Extended score at 1 year.

\section{Discussion}

In the present cohort of 163 patients assessed at a median of 3.3 months after a severe acquired brain injury, the most prevalent hormonal alterations were increased concentrations of stress-related hormones, followed by decreased gonadal and thyroid hormones, which concomitantly presented in $26 \%$ of the patients.
Similar endocrine alterations have been reported both in patients with brain injury $(4,5,6)$ and in patients with other causes of acute and severe illness (24). Although in some cases endocrine alterations may be attributed to primary or secondary injury to the hypothalamus or pituitary gland, acute severe illness involves major stress to the organism, and increased ACTH, cortisol, GH and prolactin concentrations, together with low testosterone and low triiodothyronine syndrome, have all been described as being part of important adaptive responses in the early phases following trauma, surgery and severe medical illness (24). However, distinct hormonal secretion patterns are also to be expected in the chronic phase of critical illness and in the subsequent recovery phase with the restoration of peripheral sensitivity and the loss of central drive (25). Patients included in the present study were either in the chronic phase of critical illness or in the recovery phase at the time of testing. Thus, although all of the patients still required neurorehabilitation, the cohort remained inhomogeneous, which probably explains some of the observed inter-individual differences.

A Synacthen test was routinely performed in order to diagnose hypoadrenalism. At 3 months, hypoadrenalism was only registered in two non-TBI patients. Further, up to $5 \%$ of the patients in both groups had decreased basal cortisol values, which were defined as baseline total cortisol $<2.5$ th percentile from that of healthy subjects. During critical illness, glucocorticoid secretion markedly increases and relates to stress activation of the hypothalamic-pituitary-adrenal axis (25) and reduced cortisol breakdown as a result of the suppressed expression and activity of cortisol-metabolising enzymes (26). The increase is, however, not necessarily discernible when only the total cortisol concentration is measured. In a study by Hamrahian et al. (27), nearly $40 \%$ of critically ill patients with hypoproteinaemia had subnormal total cortisol concentrations, even though their adrenal function was normal, as measured by free cortisol concentrations. Thus, lower cortisol binding globulin (CBG) concentrations (28) may have led to low cortisol concentrations in a subset of our patients, even though they responded appropriately to Synacthen stimulation. Furthermore, blood sampling was performed from 0800 to $1300 \mathrm{~h}$, which could have influenced results because of circadian hormonal rhythms. If this was the case, we may have underestimated the prevalence of elevated basal cortisol concentrations rather than overestimating them.

It is noteworthy that one-third of the patients in the present cohort had a $30 \mathrm{~min}$ cortisol concentration above that expected in healthy people, which suggests a 
Table 2 Distribution of scores in traumatic brain injury (TBI) and non-TBI patients. The table presents the number of patients with baseline and/or discharge, as well as follow-up Functional Independence Measure total scores and Glasgow Outcome Scale-Extended scores. Both scores were further divided in scoring subgroups depending on the actual level of the patients' functioning.

\begin{tabular}{|c|c|c|c|c|c|c|}
\hline \multirow[b]{2}{*}{ Time of measurement } & \multicolumn{3}{|c|}{ TBI } & \multicolumn{3}{|c|}{ Non-TBI } \\
\hline & Admission & Discharge & $\begin{array}{l}\text { One-year } \\
\text { follow-up }\end{array}$ & Admission $^{\mathrm{b}}$ & Discharge $^{b}$ & $\begin{array}{l}\text { One-year } \\
\text { follow-up }\end{array}$ \\
\hline \multicolumn{7}{|l|}{ Functional Independence Measure } \\
\hline $\begin{array}{l}\text { Complete dependency } \\
\text { (score: } 18-55)\end{array}$ & $104 / 111(94 \%)$ & $45 / 111(41 \%)$ & $23 / 93(25 \%)^{d}$ & $48 / 51(94 \%)$ & $24 / 50(48 \%)$ & $10 / 40(25 \%)^{d}$ \\
\hline $\begin{array}{l}\text { Moderate dependency } \\
\text { (score: } 56-74)\end{array}$ & $3 / 111(3 \%)$ & $8 / 111(7 \%)$ & $5 / 93(5 \%)$ & $0 / 51(0 \%)$ & $2 / 50(4 \%)$ & $2 / 40(5 \%)$ \\
\hline $\begin{array}{l}\text { Low dependency to complete } \\
\left.\text { independency }{ }^{\mathrm{c}} \text { (score: } 75-126\right)\end{array}$ & $4 / 111(3 \%)$ & $58 / 111(52 \%)$ & $65 / 93(70 \%)^{d}$ & $3 / 51(6 \%)$ & $24 / 50(48 \%)$ & $28 / 40(70 \%)^{d}$ \\
\hline \multicolumn{7}{|l|}{ Glasgow Outcome Scale-Extended } \\
\hline $\begin{array}{l}\text { Coma to upper severe disability } \\
\text { (score: } 1-4)\end{array}$ & Not done & $80 / 111(72 \%)$ & $47 / 93(51 \%)$ & Not done & $44 / 51(86 \%)$ & $25 / 40(62.5 \%)$ \\
\hline $\begin{array}{l}\text { Lower moderate disability to upper } \\
\text { good recovery (score: } 5-8 \text { ) }\end{array}$ & Not done & $31 / 111(28 \%)$ & $46 / 93(49 \%)^{e}$ & Not done & $7 / 51(14 \%)$ & $15 / 40(37.5 \%)$ \\
\hline
\end{tabular}

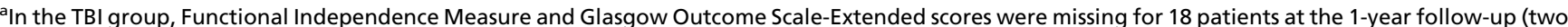
patients died before attending the follow-up visit, one patient attended the visit but that patient's score was not recorded, and 15 patients did not attend the follow-up visit for unknown reasons).

${ }^{b}$ In the non-TBI group, Functional Independence Measure scores were missing for one patient at admission, two patients at discharge, and 12 patients at the 1-year follow-up visit (three patients died before attending the follow-up visit, one patient attended the follow-up visit but that patient's score was not recorded, and eight patients did not attend the follow-up visit for unknown reasons), whereas Glasgow Outcome Scale-Extended scores were missing for one patient at discharge and for the same number of patients at the 1-year follow-up as described for the Functional Independence Measure scores.

'Three scoring groups (scores in parentheses) represent the actual need for help in performing basic daily activities. Patients in the lowest scoring group were completely dependent on assistance during the day. On the contrary, patients in the highest scoring group needed less assistance.

${ }^{\mathrm{d}}$ A significant number of patients switched from the complete dependency group (at admission) to the low dependency/complete independency group (at the 1-year follow-up) $(P<0.01)$.

${ }^{\mathrm{e}} \mathrm{A}$ significant number of patients switched from the lower-scoring group (at discharge) to the higher-scoring group (at the 1 -year follow-up) $(P<0.05)$.

prolonged stress response with glucocorticoid hyperactivation. Unfortunately, neither ACTH nor CBG were measured, and thus no correlations between total and free cortisol concentrations could be made. Finally, the documented use of the Synacthen test is apt for the assessment of hypo- and not hyperactivation of the adrenal gland. The original purpose of the study was to assess hypofunction; thus, measurements of such factors as 24-h urine cortisol, which would have been preferable for assessing hyperactivation (29), were not included.

Increased prolactin was the most commonly observed endocrine alteration. Hyperprolactinaemia is a wellknown phenomenon in the acute phase of a brain injury that may persist in $31 \%$ of patients during rehabilitation (30), and it was reported in up to $47 \%$ of the TBI patients who had other endocrine failures during long-term follow-up (31). Although hyperprolactinaemia may indicate pituitary stalk and/or hypothalamic injury, prolactin is one of the first hormones to increase in acute phases of severe illness, whereas prolactin is no longer as high in chronic phases (24).

In acute and chronic phases of critical illness, IGF1 concentrations tended to be low (32). However, Kopczak et al. (33) showed that $14 \%$ of their patients had elevated
IGF1, whereas $6 \%$ had reduced IGF1 concentrations, as measured at a median of 7 weeks after the TBI or subarachnoid haemorrhage. This was in keeping with our data of commonly registered elevated IGF1. However, it is not clear whether increased IGF1 should be considered part of neuroprotection (34) or as a result of the insulin resistance and hyperglycaemia that are commonly observed during prolonged critical illness (35). A concomitant assessment of GH concentrations might have clarified this further.

Endocrine alterations similar to secondary hypogonadism and hypothyroidism were observed in 23 and 11\% of the patients respectively. Similar findings have previously been observed in patients with brain injury $(4,5,11)$ but also in patients with critical illness resulting from other causes and without a history of acquired brain injury (24). It is well established that prolonged critical illness alters the physiology of the reproductive axis with decreased testosterone concentrations and reduced or low to normal gonadotrophins (36); these patterns have also been observed for the thyroid axis (24). The most likely explanation for these endocrine alterations in severe prolonged illness was suggested to be impairment of the pulsatile component of pituitary hormones, such as the gonadal axis (24), but it may also involve reduced 
hypothalamic stimulation of trophic hormones, which in turn leads to reduced stimulation of the target organ (25).

Because secondary hypogonadism and hypothyroidism were present concomitant with elevated stress hormones in $26 \%$ of the patients, we suggest that these endocrine alterations were a result of the prolonged stress response in the period after the severe brain injury and not a result of hypothalamo-pituitary injury.

Some recent outcome studies have indicated that posttraumatic hypopituitarism has clinical significance because it impacts both health-related quality of life and lipid status (17), but the findings have been inconsistent $(20,37)$. Ulfarsson et al. (37) tested 51 patients with severe TBI for 2-10 years after the initial trauma, which is longer than the present study, but they showed no relationship between hypopituitarism and worse psychological, cognitive or motor functioning in the longer term. In the present study, both secondary hypogonadism and increased stress hormones were related to worse 1-year follow-up scores on the Functional Independence Measure, which is well known and widely used in rehabilitation settings. A recent non-randomised retrospective study in TBI patients suggested that reduced testosterone levels at admission were related to lower admission scores, less change in scores on the Functional Independence Measure and longer hospitalisation (21). Other authors have suggested that hypogonadism may limit the pace of recovery during rehabilitation (22) because of the well-known effects of testosterone on muscle mass and strength, cognition and perception of the quality of life. Whether the same pattern of action can be applied to people with acquired severe brain injuries remains to be investigated. Kopczak et al. (33) went a step further and treated 13 male patients after TBI or subarachnoid haemorrhage with testosterone replacement. The Barthel index (a measurement of independence and recovery) and haemoglobin concentrations before and after treatment were measured, and haemoglobin had increased in four patients in 4 weeks $>2 \mathrm{~g} / \mathrm{dl}$ $(1.24 \mathrm{mmol} / \mathrm{l})$, which was a surrogate sign of testosterone replacement benefit (33). However, the distinction between primary and secondary hypogonadism was unclear, because gonadotrophins were not measured. Further studies are needed to predict if male patients diagnosed with hypogonadism after an acquired brain injury, irrespective of its cause, would achieve a better neurorehabilitation outcome with testosterone treatment.

Low Glasgow Outcome Scale-Extended scores were significantly improved from discharge to the 1-year follow-up, which implies that more patients became capable of returning to normal activities both inside and outside of their homes. However, low Glasgow Outcome Scale-Extended scores were more frequent in patients with reduced gonadal and/or thyroid hormones and increased stress hormones, whereas only increased stress hormones remained independently related to a worse Glasgow Outcome Scale-Extended score at the 1-year follow-up. Santarsieri et al. (38) recently measured cortisol in the cerebrospinal fluid of the TBI patients and showed persistently elevated cortisol during the first 6 days after trauma, which was associated with poorer outcomes, as measured by Glasgow Outcome Scale scores and Functional Independence Measure cognitive scores 6 months after the injury. Recently, pituitary abnormalities in $27 \%$ of patients 3-6 months after a subarachnoid haemorrhage were significantly related to lower median Glasgow Outcome Scale scores at follow-up, with more prevalent endocrine abnormalities occurring when bleeding sites were closer to the hypothalamus (39). In contrast to the present and previously mentioned studies, other authors did not find an association between endocrine alterations and Glasgow Outcome Scale-Extended score measures $(37,40)$ or other quality-of-life questionnaires $(18,20)$.

Some limitations of the present study should be addressed. GH stimulation tests were not part of the routine screening programme at the neurorehabilitation centre, and GH measurements are thus not available, although they could have potentially clarified the significance of the observed changes in IGF1 values. BMI has been shown to influence both GH and IGF1, the latter being decreased with BMI exceeding $32.5 \mathrm{~kg} / \mathrm{m}^{2}$ as well as with low BMI (41). Unfortunately, at the time of testing, BMI was not available for our patients, and we could thus not correct for it. Another limitation is the aetiologic difference with variable prognoses and influence on the Functional Independence Measure score. However, the number of patients within each aetiologic subgroup was too small for subgroup analyses. Although the patients in the present study represented a heterogeneous group, especially among the non-TBI patients, the vast majority had very severe trauma, which was indicated by very low physical and cognitive scores at the time of admission to neurorehabilitation, $\sim 3$ weeks after the initial trauma. Finally, the influence of medications such as antidepressants, antipsychotics and antiemetics could be additional factors for the observed frequency of such conditions as hyperprolactinaemia.

In the present study, hormonal assessment was performed $\sim 3$ months after injury. The development of post-traumatic hypopituitarism later than 3 months after injury has been suggested (6). It remains speculative, however, if such new deficiencies would have affected 
our data. The insufficiencies reported in the present study were retested by repeat measurement $\sim 12$ months after the injury (D Marina, M Klose and U Feldt-Rasmussen, unpublished data). Most had resolved, and none of the patients had any replacements initiated after the 3-month testing.

In conclusion, the present study suggested that endocrine alterations similar to secondary hypogonadism and hypothyroidism at a median of 3.3 months after an acquired severe brain injury most probably reflected a prolonged stress response rather than brain damage per se. Accordingly, not only secondary hypogonadism and hypothyroidism but also elevated stress hormones were related to worse 1-year functional outcomes.

Thus, we suggest that post-brain injury endocrine alterations are not necessarily a consequence of hypothalamic or pituitary damage; rather, they may represent physiological stress adaptations to acute critical illness, with a subsequent prolonged stress response at least 3 months post-injury. Therefore, routine pituitary assessment in the early phases (e.g. within a year) after acquired brain injury should be limited to patients that present with typical clinical features suggestive of hypopituitarism $(4,7)$.

\section{Supplementary data}

This is linked to the online version of the paper at http://dx.doi.org/10.1530/ EJE-14-1152.

\section{Declaration of interest}

$U$ Feldt-Rasmussen received unrestricted research and travel grants and speaker's fees from Novartis and Ipsen, research grants, speaker's fees and advisory board honoraria from Pfizer and a research grant from Arvid Nilsson's Fund. M Klose received an unrestricted research grant from Novo Nordic and speaker fees from Pfizer and Novartis. D Marina, A Nordenbo and A Liebach have nothing to disclose.

\section{Funding}

This research did not receive any specific grant from any funding agency in the public, commercial or not-for-profit sector

\section{Author contribution statement}

D Marina wrote the paper; A Nordenbo and A Liebach were supervisors during the whole period of the study and collected the data; M Klose and $U$ Feldt-Rasmussen initiated the study and supervised all aspects of the process; all authors contributed to the writing of the paper.

\section{Acknowledgements}

U Feldt-Rasmussen's research salary was supported by a grant from Arvid Nilsson's fund. We thank Lars Westergaard for his assistance in collecting data and constructing the patient database used in the present study.

\section{References}

1 Greenwald BD, Burnett DM \& Miller MA. Congenital and acquired brain injury. 1. Brain injury: epidemiology and pathophysiology. Archives of Physical Medicine and Rehabilitation 200384 S3-S7. (doi:10.1053/apmr.2003.50052)

2 Hyder AA, Wunderlich CA, Puvanachandra P, Gururaj G \& Kobusingye OC. The impact of traumatic brain injuries: a global perspective. NeuroRehabilitation 200722 341-353.

3 Schneider HJ, Kreitschmann-Andermahr I, Ghigo E, Stalla GK \& Agha A Hypothalamopituitary dysfunction following traumatic brain injury and aneurysmal subarachnoid hemorrhage: a systematic review. Journal of the American Medical Association 2007298 1429-1438. (doi:10.1001/jama.298.12.1429)

4 Klose M, Juul A, Struck J, Morgenthaler NG, Kosteljanetz M \& FeldtRasmussen U. Acute and long-term pituitary insufficiency in traumatic brain injury: a prospective single-centre study. Clinical Endocrinology 200767 598-606. (doi:10.1111/j.1365-2265.2007.02860.x)

5 Schneider HJ, Schneider M, Saller B, Petersenn S, Uhr M, Husemann B, von Rosen F \& Stalla GK. Prevalence of anterior pituitary insufficiency 3 and 12 months after traumatic brain injury. European Journal of Endocrinology 2006154 259-265. (doi:10.1530/eje.1.02071)

6 Tanriverdi F, Senyurek H, Unluhizarci K, Selcuklu A, Casanueva FF \& Kelestimur F. High risk of hypopituitarism after traumatic brain injury: a prospective investigation of anterior pituitary function in the acute phase and 12 months after trauma. Journal of Clinical Endocrinology and Metabolism 200691 2105-2111. (doi:10.1210/jc.2005-2476)

7 Klose M, Juul A, Poulsgaard L, Kosteljanetz M, Brennum J \& FeldtRasmussen U. Prevalence and predictive factors of post-traumatic hypopituitarism. Clinical Endocrinology 200767 193-201. (doi:10.1111/ j.1365-2265.2007.02860.x)

8 Klose M, Stochholm K, Janukonyte J, Lehman CL, Frystyk J, Andersen M, Laurberg P, Christiansen JS \& Feldt-Rasmussen U. Prevalence of posttraumatic growth hormone deficiency is highly dependent on the diagnostic set-up: results from The Danish National Study on Posttraumatic Hypopituitarism. Journal of Clinical Endocrinology and Metabolism 201499 101-110. (doi:10.1210/jc.2013-2397)

9 van der Eerden AW, Twickler MT, Sweep FC, Beems T, Hendricks HT, Hermus AR \& Vos PE. Should anterior pituitary function be tested during follow-up of all patients presenting at the emergency department because of traumatic brain injury? European Journal of Endocrinology 2010162 19-28. (doi:10.1530/EJE-09-0436)

10 Kokshoorn NE, Wassenaar MJ, Biermasz NR, Roelfsema F, Smit JW, Romijn JA \& Pereira AM. Hypopituitarism following traumatic brain injury: prevalence is affected by the use of different dynamic tests and different normal values. European Journal of Endocrinology 2010162 11-18. (doi:10.1530/EJE-09-0601)

11 Aimaretti G, Ambrosio MR, Di Somma C, Fusco A, Cannavo S, Gasperi M, Scaroni C, De Marinis L, Benvenga S, degli Uberti EC et al. Traumatic brain injury and subarachnoid haemorrhage are conditions at high risk for hypopituitarism: screening study at 3 months after the brain injury. Clinical Endocrinology 200461 320-326. (doi:10.1111/ j.1365-2265.2004.02094.x

12 Hana V, Kosak M, Masopust V, Netuka D, Lacinova Z, Krsek M, Marek J \& Pecen L. Hypothalamo-pituitary dysfunction in patients with chronic subdural hematoma. Physiological Research 201261 161-167.

13 Bondanelli M, Ambrosio MR, Carli A, Bergonzoni A, Bertocchi A, Zatelli MC, Ceruti S, Valle D, Basaglia N \& degli Uberti EC. Predictors of pituitary dysfunction in patients surviving ischemic stroke. Journal of Clinical Endocrinology and Metabolism 201095 4660-4668. (doi:10.1210/ jc.2010-0611)

14 Klose M, Brennum J, Poulsgaard L, Kosteljanetz M, Wagner A \& FeldtRasmussen U. Hypopituitarism is uncommon after aneurysmal subarachnoid haemorrhage. Clinical Endocrinology 201073 95-101. (doi:10.1111/j.1365-2265.2010.03791.x) 
15 Gardner CJ, Javadpour M, Stoneley C, Purthuran M, Biswas S, Daousi C, Macfarlane IA \& Cuthbertson DJ. Low prevalence of hypopituitarism after subarachnoid haemorrhage using confirmatory testing and with BMI-specific growth hormone cut-off levels. European Journal of Endocrinology 2012168 473-481. (doi:10.1530/EJE-12-0849)

16 Bavisetty S, Bavisetty S, McArthur DL, Dusick JR, Wang C, Cohan P, Boscardin WJ, Swerdloff R, Levin H, Chang DJ et al. Chronic hypopituitarism after traumatic brain injury: risk assessment and relationship to outcome. Neurosurgery 200862 1080-1093. (doi:10.1227/01.neu.0000325870.60129.6a)

17 Klose M, Watt T, Brennum J \& Feldt-Rasmussen U. Posttraumatic hypopituitarism is associated with an unfavorable body composition and lipid profile, and decreased quality of life 12 months after injury. Journal of Clinical Endocrinology and Metabolism 200792 3861-3868. (doi:10.1210/jc.2007-0901)

18 Wachter D, Gundling K, Oertel MF, Stracke H \& Boker DK. Pituitary insufficiency after traumatic brain injury. Journal of Clinical Neuroscience 200916 202-208. (doi:10.1016/j.jocn.2008.01.009)

19 Bondanelli M, Ambrosio MR, Cavazzini L, Bertocchi A, Zatelli MC, Carli A, Valle D, Basaglia N \& Uberti EC. Anterior pituitary function may predict functional and cognitive outcome in patients with traumatic brain injury undergoing rehabilitation. Journal of Neurotrauma 200724 1687-1697. (doi:10.1089/neu.2007.0343)

20 Pavlovic D, Pekic S, Stojanovic M, Zivkovic V, Djurovic B, Jovanovic V, Miljic N, Medic-Stojanoska M, Doknic M, Miljic D et al. Chronic cognitive sequelae after traumatic brain injury are not related to growth hormone deficiency in adults. European Journal of Neurology 201017 696-702. (doi:10.1111/j.1468-1331.2009.02910.x)

21 Young TP, Hoaglin HM \& Burke DT. The role of serum testosterone and TBI in the in-patient rehabilitation setting. Brain Injury 200721 645-649. (doi:10.1080/02699050701210426)

22 Carlson NE, Brenner LA, Wierman ME, Harrison-Felix C, Morey C, Gallagher S \& Ripley D. Hypogonadism on admission to acute rehabilitation is correlated with lower functional status at admission and discharge. Brain Injury 200923 336-344. (doi:10.1080/ 02699050902788535)

23 Klose M, Lange M, Rasmussen AK, Skakkebaek NE, Hilsted L, Haug E, Andersen M \& Feldt-Rasmussen U. Factors influencing the adrenocorticotropin test: role of contemporary cortisol assays, body composition, and oral contraceptive agents. Journal of Clinical Endocrinology and Metabolism 200792 1326-1333. (doi:10.1210/jc.2006-1791)

24 Van Den Berghe G. Endocrine evaluation of patients with critical illness. Endocrinology and Metabolism Clinics of North America 200332 385-410. (doi:10.1016/S0889-8529(03)00005-7)

25 Boonen E \& Van Den Berghe G. Endocrine responses to critical illness: novel insights and therapeutic implications. Journal of Clinical Endocrinology and Metabolism 201499 1569-1582. (doi:10.1210/ jc.2013-4115)

26 Boonen E, Vervenne H, Meersseman P, Andrew R, Mortier L, Declercq PE, Vanwijngaerden YM, Spriet I, Wouters PJ, Vander PS et al. Reduced cortisol metabolism during critical illness. New England Journal of Medicine 2013368 1477-1488. (doi:10.1056/ NEJMoa1214969)

27 Hamrahian AH, Oseni TS \& Arafah BM. Measurements of serum free cortisol in critically ill patients. New England Journal of Medicine 2004 350 1629-1638. (doi:10.1056/NEJMoa020266)
28 Beishuizen A, Thijs LG \& Vermes I. Patterns of corticosteroid-binding globulin and the free cortisol index during septic shock and multitrauma. Intensive Care Medicine 200127 1584-1591. (doi:10.1007/ s001340101073)

29 Carroll TB \& Findling JW. The diagnosis of Cushing's syndrome. Reviews in Endocrine \& Metabolic Disorders 201011 147-153. (doi:10.1007/s11154-010-9143-3)

30 Agha A, Rogers B, Mylotte D, Taleb F, Tormey W, Phillips J \& Thompson CJ. Neuroendocrine dysfunction in the acute phase of traumatic brain injury. Clinical Endocrinology $200460584-591$. (doi:10.1111/j.1365-2265.2004.02023.x)

31 Benvenga S, Campenni A, Ruggeri RM \& Trimarchi F. Clinical review 113: Hypopituitarism secondary to head trauma. Journal of Clinical Endocrinology and Metabolism 200085 1353-1361. (doi:10.1210/ jcem.85.4.6506)

32 Mesotten D \& Van Den Berghe G. Changes within the growth hormone/insulin-like growth factor I/IGF binding protein axis during critical illness. Endocrinology and Metabolism Clinics of North America 200635 793-805, ix-x. (doi:10.1016/j.ecl.2006.09.010)

33 Kopczak A, Kilimann I, von Rosen F, Krewer C, Schneider HJ, Stalla GK $\&$ Schneider M. Screening for hypopituitarism in 509 patients with traumatic brain injury or subarachnoid hemorrhage. Journal of Neurotrauma 201431 99-107. (doi:10.1089/neu.2013.3002)

34 Rubovitch V, Edut S, Sarfstein R, Werner H \& Pick CG. The intricate involvement of the insulin-like growth factor receptor signaling in mild traumatic brain injury in mice. Neurobiological Disorders 201038 299-303.

35 Van den Berghe G. How does blood glucose control with insulin save lives in intensive care? Journal of Clinical Investigation $2004 \mathbf{1 1 4}$ 1187-1195. (doi:10.1172/JCI23506)

36 Spratt DI. Altered gonadal steroidogenesis in critical illness: is treatment with anabolic steroids indicated? Best Practice \& Research. Clinical Endocrinology \& Metabolism 200115 479-494. (doi:10.1053/ beem.2001.0165)

37 Ulfarsson T, Gudnason GA, Rosen T, Blomstrand C, Stibrant SK, Lundgren-Nilsson A \& Nilsson M. Pituitary function and functional outcome in adults after severe traumatic brain injury: the long-term perspective. Journal of Neurotrauma 201330 271-280. (doi:10.1089/neu. 2012.2494)

38 Santarsieri M, Niyonkuru C, McCullough E, Dobos J, Dixon CE, Berga S $\&$ Wagner AK. CSF cortisol and progesterone profiles and outcomes prognostication after severe TBI. Journal of Neurotrauma 201431 699-712. (doi:10.1089/neu.2013.3177)

39 Kronvall E, Valdemarsson S, Saveland H \& Nilsson OG. Pituitary dysfunction after aneurysmal subarachnoid hemorrhage is associated with impaired early outcome. World Neurosurgery 201381 529-537. (doi:10.1016/j.wneu.2013.10.038)

40 Wagner J, Dusick JR, McArthur DL, Cohan P, Wang C, Swerdloff R, Boscardin WJ \& Kelly DF. Acute gonadotroph and somatotroph hormonal suppression after traumatic brain injury. Journal of Neurotrauma 201027 1007-1019. (doi:10.1089/neu.2009.1092)

41 Schneider HJ, Saller B, Klotsche J, Marz W, Erwa W, Wittchen HU \& Stalla GK. Opposite associations of age-dependent insulin-like growth factor-I standard deviation scores with nutritional state in normal weight and obese subjects. European Journal of Endocrinology $2006 \mathbf{1 5 4}$ 699-706. (doi:10.1530/eje.1.02131)

Received 28 December 2014

Revised version received 10 March 2015

Accepted 30 March 2015 